\title{
A comparative study of acellular nerve xenografts and allografts in repairing rat facial nerve defects
}

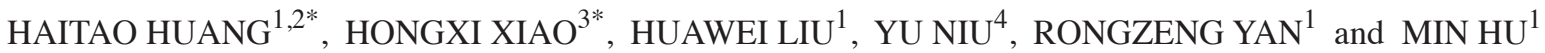 \\ ${ }^{1}$ Department of Stomatology, Chinese PLA General Hospital, Beijing 100853; ${ }^{2}$ Department of Stomatology, \\ The 1st Affiliated Hospital of Dalian Medical University, Dalian, Liaoning 116011; ${ }^{3}$ Department of Stomatology, \\ The 309th Hospital of Chinese PLA, Beijing 100083; ${ }^{4}$ Department of Maxillofacial Surgery, \\ The Second Hospital of Liaohe Oil Field, Panjin, Liaoning 124010, P.R. China
}

Received October 1, 2014; Accepted June 26, 2015

DOI: $10.3892 / \mathrm{mmr} .2015 .4123$

\begin{abstract}
Acellular nerves are composed of a basal lamina tube, which retains sufficient bioactivity to promote axon regeneration, thereby repairing peripheral nerve gaps. However, the clinical application of acellular allografts has been restricted due to its limited availability. To investigate whether xenografts, a substitute to allograft acellular nerves in abundant supply, could efficiently promote nerve regeneration, rabbit and rat acellular nerve grafts were used to reconstruct $1 \mathrm{~cm}$ defects in Wistar rat facial nerves. Autologous peroneal nerve grafts served as a positive control group. A total of 12 weeks following the surgical procedure, the axon number, myelinated axon number, myelin sheath thickness, and nerve conduction velocity of the rabbit and rat-derived acellular nerve grafts were similar, whereas the fiber diameter of the rabbit-derived acellular xenografts decreased, as compared with those of rat-derived acellular allografts. Autografts exerted superior effects on nerve regeneration; however, no significant difference was observed between the axon number in the autograft group, as compared with the two acellular groups. These results suggested that autografts perform better than acellular nerve grafts, and chemically extracted acellular allografts and xenografts have similar effects on the regeneration of short facial nerve defects.
\end{abstract}

\section{Introduction}

Segmental peripheral nerve defects are a clinical problem that may occur following trauma or tumor resection, and the nerve

Correspondence to: Dr Min Hu, Department of Stomatology, Chinese PLA General Hospital, 28 Fuxing Road, Beijing 100853, P.R. China

E-mail: minhu48@vip.163.com

*Contributed equally

Key words: nerve graft, nerve regeneration, acellular nerve, xenograft, peripheral nerve gap is usually too large to allow primary end-to-end nerve coaptation in the absence of tension. Under these conditions, reconstruction of the peripheral nerve gap with grafts is necessary, in order to avoid the complete loss of motor function and sensation of the affected area. For the past 100 years, reconstruction of the peripheral nerve gap has been achieved with autografts, as these offered the highest chance of functional recovery (1). However, limited amounts of donor nerve, as well as deficits in the donor area following nerve graft harvest restrict the clinical application of nerve gap reconstruction using autografts (2). Therefore, current research has focused on identifying an alternative to autogenous nerve grafts.

Allografts have been suggested as an effective alternative to autogenous nerve grafts in the repair of nerve gaps, due to their similar physical, chemical, and mechanical properties. A previous study reported that processed allograft has similar effects on nerve function recovery, as compared with autografts in certain animal studies (3). However, the limited number of donors is unable to meet the clinical requirements of the increasing number of recipients and commercial allogeneic nerve grafts are not yet available. Therefore, xenografts are now being considered as substitutes to allografts and autografts, due to the large number of donors.

Xenografts have limitations when used as transplants to repair nerve gaps, with the most significant limitation being immune rejection caused by donor antigens (4). Immune rejection may impair xenograft nerve regeneration, in a similar manner to allografts (5). Two primary methods are used to achieve graft tolerance, one of which is the treatment of recipients with immunosuppressive drugs, such as FK506 or cyclosporin A (6); and the other is processing of the nerve prior to grafting in order to render it less immunogenic (7). The systemic administration of immunosuppressive drugs is necessary for whole organ transplants, but is not an efficient solution for peripheral nerve transplants due to the side effects caused by the medication, which may be more severe than the disease itself (8). Therefore, current research has focused on decreasing the antigenicity of xenogeneic nerves with a series of treatments prior to grafting, in order to inhibit the immune response.

A previous study reported that chemically extracted nerves were able to support axon growth in both laboratory 
and clinical settings, due to the absence of cells and myelin in the nerves, which act as major donor antigens (9). In addition, chemically extracted nerves may support axon growth by maintaining the structural integrity of the basal lamina, which has an important role in guiding regenerating axons toward their targets (10). However, the majority of studies regarding acellular nerve grafts have been conducted on allotransplantation, and little data are available on acellular nerve grafts in xenotransplantation. Furthermore, before xenograft acellular nerves may be used in clinical settings, further research is required in order to identify appropriate animal donor species, which includes the selection and matching of a suitable combination of donor grafts and host species.

In the present study, chemically extracted rabbit facial acellular nerve grafts were used to repair $1 \mathrm{~cm}$ rat facial nerve defects, and the outcome of axonal regeneration and functional restoration following nerve gap reconstruction was observed morphologically and electrophysiologically. The present study hypothesized that acellular nerve xenografts may exhibit comparable histological and functional outcomes as that of acellular nerve allografts in repairing segmental nerve defects.

\section{Materials and methods}

Donor nerve harvest. New Zealand white rabbits (weight, 2.0-2.4 kg; Center of Animal Experiments, Chinese PLA General Hospital, Beijing, China) were anesthetized by intravenous injection of 3\% pentobarbital sodium (Sangon Biotech Co., Ltd., Shanghai, China) and Wistar rats (weight, 200-220 g; Center of Animal Experiments Chinese PLA General Hospital were anesthetized by intraperitoneal injection of $10 \%$ chloral hydrate (Sangon Biotech Co., Ltd.), a postauricular incision was made, and the facial nerve was exposed. A length of $4-5 \mathrm{~cm}$ of the rabbit facial nerves were removed as xenograft nerves, and a length of $2 \mathrm{~cm}$ of rat facial nerves were removed as allograft nerves. All of the tissue specimens were washed in a distilled water bath and cryopreserved.

Facial nerve acellularization. The acellular nerves were prepared via a chemical extraction process as described in our previous study (11). The facial nerve segments from both the rats and rabbits were washed in distilled water for $12 \mathrm{~h}$, prior to being immersed in 3\% Triton X-100 solution (Beijing Zhongshan Jinqiao Biotechnology Co., Ltd., Beijing, China) for $12 \mathrm{~h}$. The nerves were then immersed in a distilled water bath for $3 \mathrm{~h}$, and transferred for a $12 \mathrm{~h}$ period into a solution of $4 \%$ sodium deoxycholate in distilled water. These steps were then repeated. Following a final wash in a distilled water bath for $3 \mathrm{~h}$, the extracted nerves were removed and placed in sterile phosphate-buffered saline $(\mathrm{pH} 7.2)$, and stored at $4^{\circ} \mathrm{C}$.

Experimental animals and study design. A total of 18 Wistar rats (weight, 200-220 g) were used. All animals were housed in a central animal care facility, where temperatures ranged between 22 and $24^{\circ} \mathrm{C}$ and humidity levels ranged between 50 and $60 \%$, with 12-hour light-dark cycles and food and water ad libitum. The rats were divided into three groups $(\mathrm{n}=6)$ : An acellular nerve allograft group, an acellular nerve xenograft group and an autologous nerve graft group.
Surgical procedure. Following intraperitoneal injection of $10 \%$ chloral hydrate $(0.4 \mathrm{ml} / 100 \mathrm{mg}$; Sangon Biotech Co., Ltd.), a postauricular incision was made on the right side of the face, and the parotid gland and facial nerve were exposed. A segment of the buccal facial nerve branch was resected leaving a $1 \mathrm{~cm}$ gap. In the allograft group, the facial nerve gap was reconstructed using an acellular facial nerve graft from another rat. In the xenograft group, the facial nerve gap was reconstructed using an acellular facial nerve graft from a rabbit. In the autograft group, an incision parallel to the femur was made, and the peroneal nerve was exposed via a gluteal muscle-splitting approach. Subsequently, the facial nerve gap was reconstructed using an autogeneic peroneal nerve graft. The donor nerves were trimmed to match the diameter and length of the facial nerve of recipients prior to grafting. The nerve grafts were anastomosed to the proximal and distal nerve stumps under an OPMI 6-SD microscope (Carl Zeiss, Oberkochen, Germany) using 9-0 nylon sutures (Shandong Sinorgmed Co., Ltd., Heze, China). The incision was sutured and disinfected using povidone iodine. All experimental procedures were approved by the Ethics Committee of the Chinese PLA general hospital.

Functional evaluation of nerve regeneration. Electrophysiological examination was conducted on the rats prior to sacrifice using an NDI-200P+ electroneurogram device (Shanghai Haishen Medical Electronic Instrument Co., Ltd., Shanghai, China), in order to detect the extent of facial nerve functional recovery. The rats were anesthetized by intraperitoneal injection of $10 \%$ chloral hydrate $(0.4 \mathrm{ml} / 100 \mathrm{mg})$, and the buccal branches of the right facial nerves and corresponding orbicularisoris muscles were exposed. Excitation electrodes were placed on the proximal end of the nerve, and recording electrodes were placed on the distal end of the graft. The proximal end was then stimulated with square waves, the action potential caused by the stimulus was recorded, and the nerve conduction velocity was calculated.

Histological and morphological evaluation of nerve regeneration

Histological staining. Hematoxylin and Eosin (HE; Beijing Zhongshan Jinqiao Biotechnology Co., Ltd.) staining was performed to observe the constitution of acellular nerves. Twelve weeks following the surgical procedure, six animals in each group were anesthetized by intraperitoneal injection of $10 \%$ chloral hydrate $(0.4 \mathrm{ml} / 100 \mathrm{mg})$, and the anastomosis site of the graft was exposed to allow observation of nerve growth. The nerve graft was then harvested and the distal part of the anatomosis site $(2 \mathrm{~mm})$ was fixed in $4 \%$ paraformaldehyde for $24 \mathrm{~h}$. Following dehydration using graded ethanol baths, the tissue sections were fixed in paraffin, and selected for histological and morphological analysis under random high power fields. Masson's trichrome staining (Shanghai Seebio Biotech Co., Ltd., Shanghai, China) was performed to observe nerve fiber regeneration and cell growth. Semi-thin $(2 \mu \mathrm{m})$ sections were then cut from the tissue sections prior to staining with toluidine blue (Shanghai Rongbo Biotech Co., Ltd., Shanghai, China) in order to measure regenerating axon count, myelinated axon count, fiber diameter, and myelin thickness. The images were captured using a BX51 light microscope (Olympus Corporation, Tokyo, Japan). 
Immunohistochemical staining. Immunohistochemistry was performed on $5 \mu \mathrm{m}$ sections to observe the distribution of Schwann cells in the regenerated nerve fibers. The slides containing the tissue sections were blocked with goat serum for 30 min (Beijing Zhongshan Jinqiao Biotech Co., Ltd.) at room temperature. The tissue sections were subsequently incubated with anti-S100 rabbit monoclonal primary antibody (1:500; cat. no. ZA-0225; Beijing Zhongshan Jinqiao Biotechnology Co., Ltd.) overnight at $4^{\circ} \mathrm{C}$. Following three washes in distilled water, the sections were incubated with biotin-labeled anti-rabbit immunoglobulin G secondary antibody (cat. no. SP-9000-D; Beijing Zhongshan Jinqiao Biotechnology Co., Ltd.) for $2 \mathrm{~h}$ at room temperature, and 3,3'-diaminobenzidine substrate (Sigma-Aldrich, St. Louis, MO, USA) was used to develop colored stains. The images were captured using an Olympus DP50 camera (Olympus Corporation) connected to a TS100 fluorescence microscope (Nikon Corporation, Tokyo, Japan). The data were analyzed using an MPLAS 500 color pathology picture analysis system (Wuhan Qingping Imaging Technology Co., Ltd., Wuhan, China).

Transmission electron microscopy (TEM). Uranyl acetate and lead citrate double staining (Qingdao Jieshi Kang Biotech Co., Ltd., Qingdao, China) were performed on ultra-thin nerve tissue sections following fixation with $3 \%$ glutaraldehyde and embedding with resin, in order to detect the ultrastructure of the regenerated nerve fibers under a Hitachi H-7000 TEM (Hitachi Co., Tokyo, Japan).

Statistical analysis. To compare the statistically significant differences among the various groups, the data were analyzed using SPSS 13.0 software (SPSS, Inc., Chicago, IL, USA). One-way analysis of variance was used to analyze the data. All experimental results were expressed as the mean \pm standard deviation. $\mathrm{P}<0.05$ was considered to indicate a statistically significant difference.

\section{Results}

Acellular nerve structure. HE staining indicated that cells and myelin were absent in both the transverse and longitudinal sections of the acellular nerves; however, the basal membrane remained intact. TEM further demonstrated that only collagen fibers, which are the principle component of the extracellular matrix (ECM), were preserved following chemical extraction decellularization, and formed a regular structure of basal lamina tubes (Fig. 1).

General observations. All of the nerve transplants exhibited adequate continuity between the proximal and distal stumps of the recipient nerves, and did not present edema. No significant inflammation was present surrounding the grafts, and only minor adherence and scar tissue formation were observed between the transplants and nearby tissues, allowing the nerve to be easily separated.

Histological observations of nerve regeneration. Masson's trichrome staining was performed 12 weeks following the surgical procedure, and indicated that in a similar manner to autografts, abundant regenerative nerve fiber bundles invaded the center of the transplants in both the allografts and xenografts. Neovascularization and a small amount of fibrous tissue hyperplasia were also observed (Fig. 2A-C). These results suggest that the regenerative axons are able to pass through the $1 \mathrm{~cm}$ acellular nerve transplants, regardless of graft type.

Toluidine blue staining. Nerve regeneration was quantitatively evaluated using histomorphometric measurements following toluidine blue staining. Both myelinated nerve fibers and non-myelinated nerve fibers were located in the cross-sections of the regenerating nerves of the three groups. However, the axons appeared more uniform in the autograft group, as compared with those of either acellular nerve graft groups (Fig. 2D-F). No significant difference in axon number was observed between the various groups ( $\mathrm{P}>0.05$; Fig. $3 \mathrm{~A}$ ), whereas the number of myelinated axons was higher in the autograft group, as compared with the xenograft and allograft groups (Fig. 3B), and a higher number of unmyelinated axons were located in the acellular xenograft and allograft nerve groups, as compared with the autograft group. Fiber diameter and myelin sheath thickness in the autograft group increased significantly, as compared with in the allograft and xenograft groups $(\mathrm{P}<0.05$; Fig. $3 \mathrm{C}$ and $\mathrm{D})$. With the exception of fiber diameter, the differences in nerve regeneration values between the allograft and the xenograft group were not statistically significant ( $\mathrm{P}>0.05$; Fig. 3).

TEM observations. In addition to myelinated and non-myelinated nerve fibers, Schwann cells were also located in the cross-sections of regenerating nerve fibers, as determined by TEM (Fig. 4A-C). Schwann cells from the host were able to migrate into the chemically processed acellular nerve grafts, in order to form myelin sheaths enclosing the regenerative axons. In addition, the autografts exhibited significantly higher myelinated axon number, myelin sheath thickness, and fiber diameter $(\mathrm{P}<0.05)$, as compared with both allografts and xenografts.

Immunohistochemical staining. Immunohistochemical staining indicated that S-100-positive cells arranged along the new nerve fibers in each of the three groups, forming a long shuttle. A higher number of positive cells were present in the autograft group, as compared with the xenograft and allograft nerve groups (Fig. 5).

Nerve conduction velocity examination. Electrophysiological analysis determined that nerve conduction velocity in the autograft group was significantly higher, as compared with the xenograft and allograft acellular nerve graft groups, 12 weeks following transplant $(\mathrm{P}<0.05)$. However, no significant differences were observed between the allograft and xenograft groups (Fig. 6).

\section{Discussion}

The structure of the nerve graft is able to influence nerve regeneration capacity, with acellular nerve grafts containing natural tubular structures that support axon ingrowth, and therefore may be used as a biomaterial to bridge peripheral nerve gaps (12-14). In addition, acellular nerve grafts contain 
A

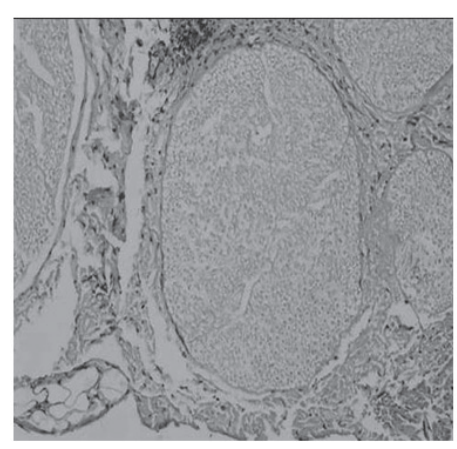

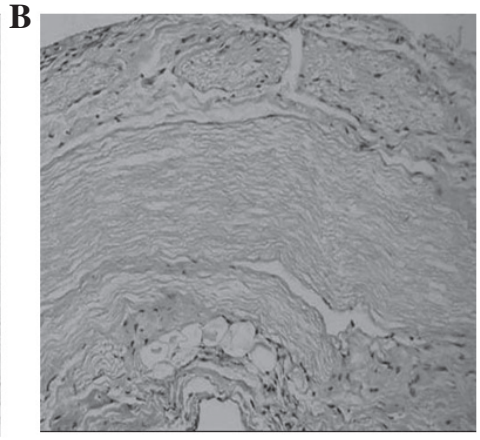

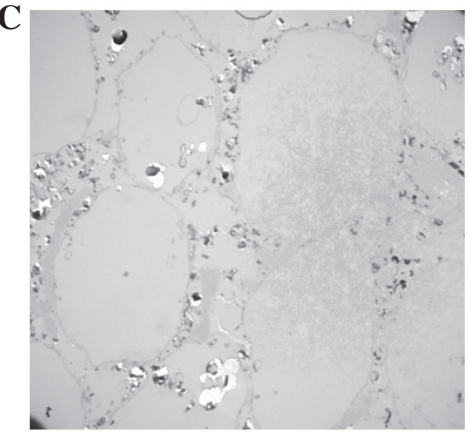

Figure 1. Morphological changes in the rabbit facial nerve following chemical acellularization via hematoxylin and eosin staining. (A) Transverse section, (B) longitudinal section, and (C) transmission electron microscopy, indicating the presence of an intact basal membrane, and absence of cellular components.
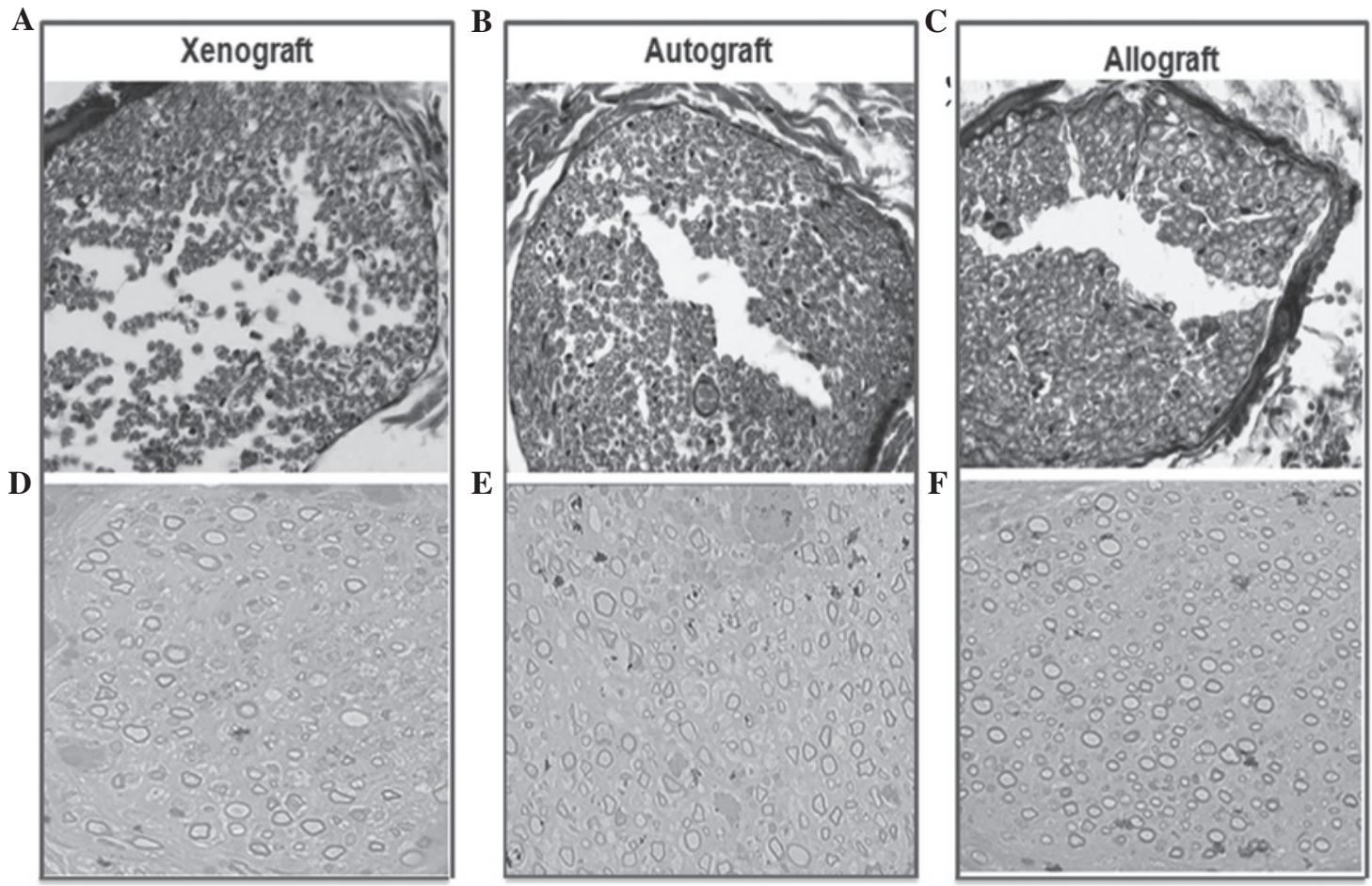

Figure 2. Histological and morphological evaluation of the distal regenerative nerve fibers 12 weeks following surgical transplantation. (A-C) Masson's trichrome staining. Abundant regenerative nerve fiber bundles invaded the center of the transplants in all three groups. Neovascularization and a small amount of fibrous tissue hyperplasia were also observed (magnification, x100). (D-F) Toluidine blue staining (magnification, x200). Both myelinated and non-myelinated nerve fibers were present in the cross-sections of regenerating nerves, and no significant differences in axon number was observed between the different groups $(\mathrm{P}>0.05)$.

components of the ECM, and are predominantly composed of laminin, heparin sulfate proteoglycan, fibronectin, and collagen type IV, which release growth factors to guide axonal elongation and promote Schwann cell migration (15-17). Due to these advantages, acellular nerve grafts have been considered an ideal alternative to autografts. Previous studies have focused on the use of acellular allografts for peripheral nerve gap reconstruction $(3,12-14)$, whereas the use of acellular xenografts has rarely been reported. However, xenografts are available in greater quantities, which would provide grafts for an increasing number of potential recipients. In the present study, HE staining and TEM demonstrated that acellular nerves derived from rabbits presented native three-dimensional basal lamina tube microstructures, and the collagen fibers of the conduit wall were arranged regularly. Both the xenografts and allografts had similar numbers of regenerative axons as the autografts, indicating that the donor ECM structure mimics the native nerve and may provide a favorable local environment for axon regeneration.

Schwann cells, endothelial cells, and macrophages from donor nerves are the principle antigens responsible for immunological rejection, which can impair nerve regeneration in xenografts (18-20). Therefore, removal of cellular components prior to grafting is essential for successful nerve regeneration. In the present study, a chemical extraction method was used to eliminate cellular antigens, and no cellular components were detected following acellular histomorphometric examination of the HE-stained nerves, as determined by TEM. As a result, no immunological rejection or inflammation was observed 12 weeks following surgical graft transplantation, 
A

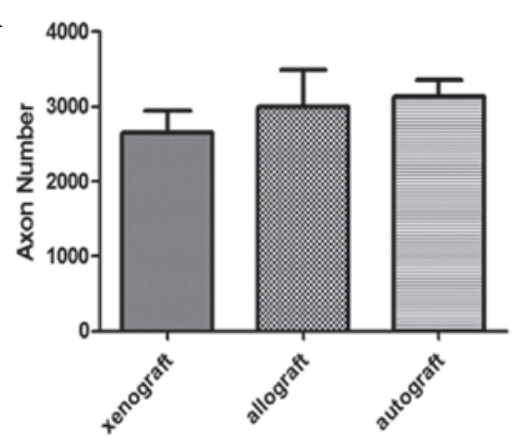

C

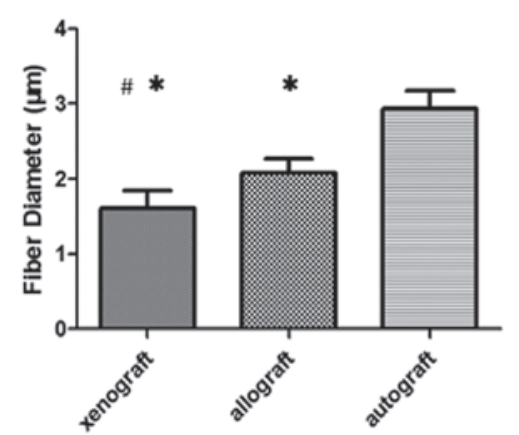

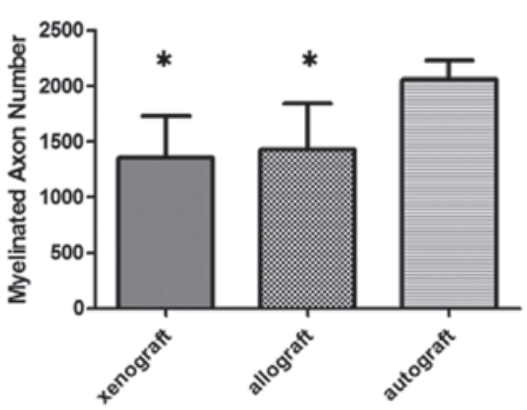

D

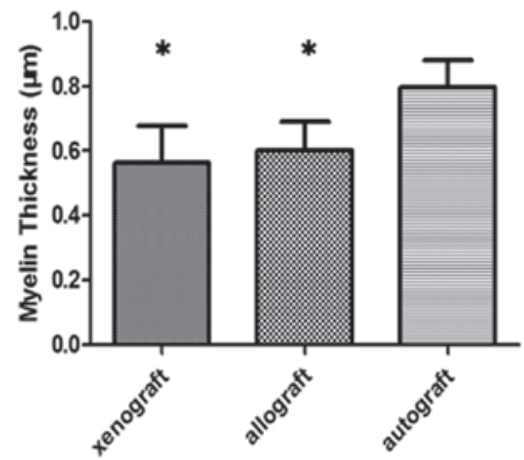

Figure 3. Quantitative analysis of distal regenerative nerve fibers 12 weeks following graft transplantation. (A) No significant differences in axon number were observed between the various groups, whereas the (B) myelinated axon number, (C) fiber diameter, and (D) myelin sheath thickness in the autograft group increased significantly, as compared with those of the allograft and xenograft acellular nerve groups. With the exception of fiber diameter, the differences in the nerve regeneration values between the allograft and xenograft groups were not statistically significant. Data were expressed as the mean \pm standard deviation. ${ }^{*} \mathrm{P}<0.05$, vs. the autograft group; ${ }^{*} \mathrm{P}<0.05$, vs. the allograft group.
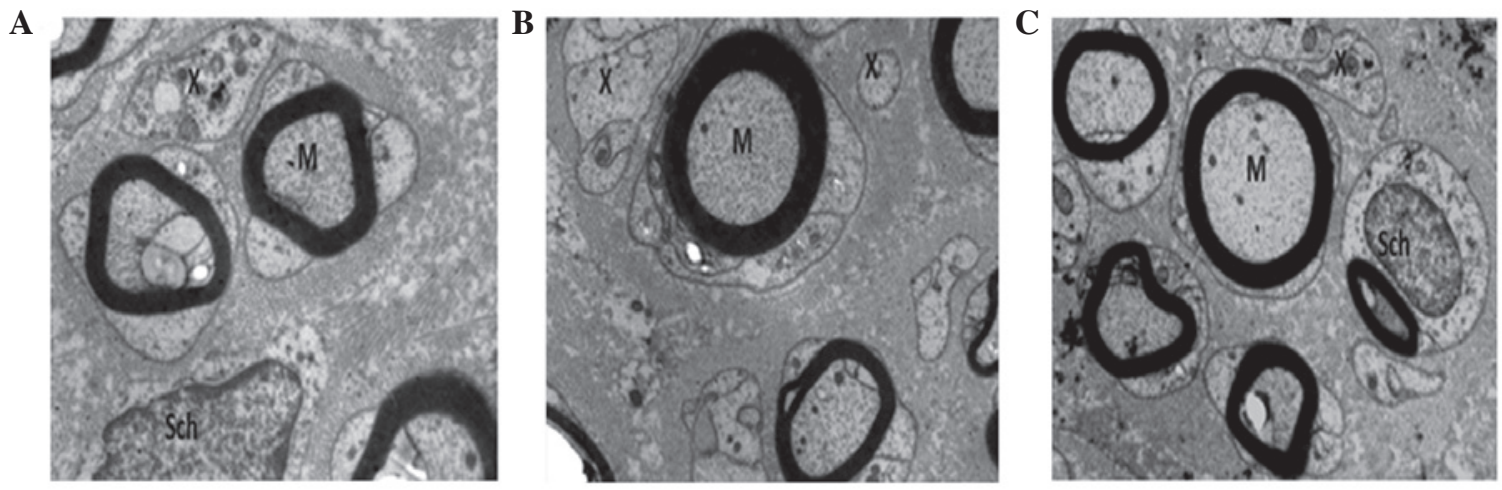

Figure 4. Transmission electron microscopy. The number of myelinated axons (M) and Schwann cells (Sch) were less in the (A) xenograft and (B) allograft acellular nerve groups, as compared with the (C) autograft group, and a higher number of unmyelinated axons (X) were located in the xenograft and allograft acellular nerve groups, as compared with the autograft group (magnification, $\mathrm{x} 8,000$ ).
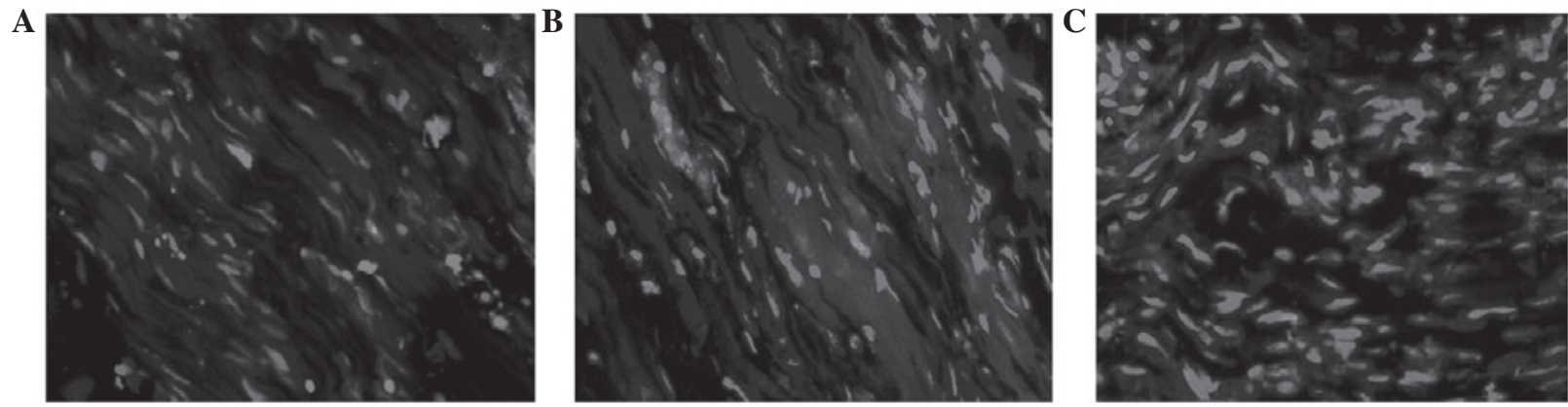

Figure 5. Immunohistochemical staining of Schwann cells in longitudinal sections of regenerative nerve fibers. (A) Acellular xenograft; (B) acellular allograft; (C) autograft. A higher number of positive cells were present in the autograft group, compared with the xenograft and allograft groups (magnification, x200). 


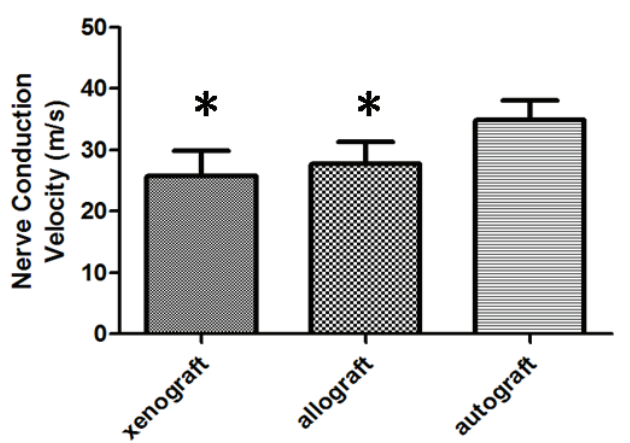

Figure 6. Nerve conduction velocity of the regenerating nerves. Nerve conduction velocity in the autograft group was markedly higher, as compared with both the acellular nerve graft groups, but no significant difference was observed between the allograft and xenograft groups. The data are expressed as the mean \pm standard deviation. ${ }^{*} \mathrm{P}<0.05$, vs. the autograft group.

demonstrating that chemically extracted nerves could result in immune tolerance to both allografts and xenografts.

Chemically-treated grafts exhibited an absence of Schwann cells, as compared with autografts, which are essential for nerve regeneration and maturation (21). During nerve regeneration, and following axon growth into the graft, Schwann cells enter the graft and reoccupy the basal lamina, resulting in the formation of myelin, which contributes to the recovery of neural function $(22,23)$. In the present study the amount of regenerative axons was similar between the acellular nerve graft groups and the autograft group; however, the number of myelinated axons, the thickness of the myelin sheath, and the fiber diameter, which represent the maturity of regenerative axons, were markedly reduced in the acellular nerve graft groups, as compared with the autograft group. The higher number of unmyelinated axons in the acellular nerve grafts may be due to the fact that the leading front of new axons in regenerating nerves are composed of non-myelinated axons (24), which remain immature and may become myelinated as Schwann cells invade the graft. Immunohistochemical staining and TEM analysis demonstrated that Schwann cells were able to migrate into regenerative axons to form myelin sheaths in both the xenograft and allograft acellular nerve groups, but a smaller number of Schwann cells were available in these two groups, as compared with the autograft group, which could reduce the speed of axonal growth and maturation. These data suggested that acellular nerves are able to support nerve fiber ingrowth within the autogenous nerve. Autograft nerves exhibited advantages, due to the fact that autogenous nerve grafts were the only grafts to contain living Schwann cells, which could increase the speed of myelin formation.

Previous studies have suggested that a deficiency in Schwann cells in the regenerated nerves of xenograft and allograft groups may partly be due to the extensive duration of the extraction process, which may reduce the number of factors that are able to aid Schwann cell migration $(22,25)$. Therefore, further studies are required in order to shorten the time taken to carry out the extraction process, thus allowing the retention of growth factors released from the ECM. In previous studies, Schwann cells $(26,27)$, and neurotrophic factors $(28,29)$ have been placed into grafts in order to improve nerve regeneration. A previous study demonstrated that grafts containing Schwann cells were able to provide a better environment for nerve regeneration (30); conversely, other studies demonstrated that acellular nerves had the same ability to support axon regeneration $(31,32)$.

In addition to histological examination, autografts also exhibited the best nerve regeneration results following electrophysiological analysis. The data indicated that nerve conduction velocity in the autograft group was markedly higher, as compared with those in the acellular nerve graft groups, however no significant differences were present between the allograft and xenograft groups, results that were concordant with those of previous histological findings. A previous study reported that there is close correlation between histomorphometric and electrophysiological analysis results (16), and increased nerve regeneration is indicative of increased functional recovery (33). Rats receiving xenografts displayed poorer functional recovery, as compared with those receiving autografts shortly following grafting, however over a longer period of time, functional recovery was similar between the two groups (9). These results suggested that xenografts and autografts lead to similar functional recovery over a suitable period of time; however, autografts are able to induce rapid functional recovery, as compared with acellular xenografts, due to the presence of better cellular support. In the present study, the data were measured 12 weeks following grafting, but in order to confirm whether Schwann cells are able to induce functional nerve recovery in autografts at earlier time points, further long-term studies are required.

The results of the present study demonstrated that chemically-treated allografts and xenografts had similar effects on nerve regeneration, including regenerative axon number, myelinated axon number, and thickness of myelin sheath, leaving only fiber diameter which was statistically different. These results are concordant with those of a previous study (34), and indicate that chemically extracted nerves have similar axon regeneration abilities when repairing a short nerve defect. However, another study reported that allografts perform better, as compared with xenografts, when repairing a $14 \mathrm{~mm}$ rat sciatic nerve defects (35). A possible cause for these differences in regeneration potential in the latter study was the use of human-derived processed nerves, whereas the present study used rabbit-derived processed nerves as xenografts to repair rat nerve defects. Animal experiments have confirmed that in order to bridge a nerve defect, xenografts from certain host species induce better nerve regeneration than others (36). However, it remains unclear why and how the xenografts from these various host species affect nerve regeneration. Therefore, when comparing these two studies, genetic differences that may affect nerve regeneration must be taken into account. Furthermore, the method of selection and matching of a suitable combination of donor grafts and host species requires further study in order to improve axonal outgrowth.

The present study had limitations insofar as the experimental model focused on a small gap defect alone. However, to repair a $1 \mathrm{~cm}$ nerve gap, chemically extracted acellular xenografts may be used as substitutes to allografts. In the future, wider gaps should be reconstructed in order to examine whether allografts and xenografts have similar effects on nerve regeneration. 
The present study demonstrated that chemically extracted acellular facial nerves no longer contained cellular material, and maintained the structural integrity of the basal lamina, which mimicked the native nerves and supported facial nerve regeneration, resulting in functional recovery both in allografts and in xenografts. However, autografts exhibited superior functional recovery and nerve regeneration, as compared with allografts and xenografts. Allografts and xenografts exhibited a similar ability to induce nerve regeneration, with the exception of fiber diameter. In conclusion, chemically extracted acellular allografts and xenografts have comparable effects on reconstruction of short facial nerve defects.

\section{Acknowledgements}

This study was funded by a grant from the National Natural Science Foundation of China (grant. no. 30872898) and a grant from Beijing Municipal National Science Foundation (grant. no. 7132173).

\section{References}

1. Millesi H: Techniques for nerve grafting. Hand Clin 16: 73-91, 2000.

2. Terzis JK, Sun DD and Thanos PK: Historical and basic science review: Past, present, and future of nerve repair. J Reconstr Microsurg 13: 215-225, 1997.

3. Zhong H, Chen B, Lu S, Zhao M, Guo Y and Hou S: Nerve regeneration and functional recovery after a sciatic nerve gap is repaired by an acellular nerve allograft made through chemical extraction in canines. J Reconstr Microsurg 23: 479-487, 2007.

4. Lu LJ, Sun JB, Liu ZG, Gong X, Cui JL and Sun XG: Immune responses following mouse peripheral nerve xenotransplantation in rats. J Biomed Biotechnol 2009: 412598, 2009.

5. Platt JL: A perspective on xenograft rejection and accommodation. Immunol Rev 141: 127-149, 1994.

6. Udina E, Voda J, Gold BG and Navarro X: Comparative dose-dependence study of FK506 on transected mouse sciatic nerve repaired by allograft or xenograft. J Peripher Nerv Syst 8: 145-154, 2003

7. Evans PJ, Midha R and Mackinnon SE: The peripheral nerve allograft: A comprehensive review of regeneration and neuroimmunology. Prog Neurobiol 43: 187-233, 1994.

8. Scherer MN, Banas B, Mantouvalou K, Schnitzbauer A, Obed A, Krämer BK and Schlitt HJ: Current concepts and perspectives of immunosuppression in organ transplantation. Langenbecks Arch Surg 392: 511-523, 2007.

9. Accioli De Vaconcellos ZA, Duchossoy Y, Kassar-Duchossoy L and Mira JC: Experimental median nerve repair by fresh or frozen nerve autografts and xenografts. Ann Chir Main Memb Super 18: 74-84, 1999.

10. Hudson TW, Liu SY and Schmidt CE: Engineering an improved acellular nerve graft via optimized chemical processing. Tissue Eng 10: 1346-1358, 2004.

11. Hu M, Zhang L, Niu Y, Xiao H, Tang P and Wang Y: Repair of whole rabbit facial nerve defects using facial nerve allografts J Oral Maxillofac Surg 68: 2196-2206, 2010.

12. Karabekmez FE, Duymaz A and Moran SL: Early clinical outcomes with the use of decellularized nerve allograft for repair of sensory defects within the hand. Hand (NY) 4 : 245-249, 2009.

13. Brooks DN, Weber RV, Chao JD, Rinker BD, Zoldos J, Robichaux MR, Ruggeri SB, Anderson KA, Bonatz EE, Wisotsky SM, et al: Processed nerve allografts for peripheral nerve reconstruction: A multicenter study of utilization and outcomes in sensory, mixed, and motor nerve reconstructions. Microsurgery 32: 1-14, 2012.

14. Cho MS, Rinker BD, Weber RV, Chao JD, Ingari JV, Brooks $\mathrm{D}$ and Buncke GM: Functional outcome following nerve repair in the upper extremity using processed nerve allograft. J Hand Surg Am 37: 2340-2349, 2012.
15. Rovak JM, Bishop DK, Boxer LK, Wood SC, Mungara AK and Cederna PS: Peripheral nerve transplantation: The role of chemical acellularization in eliminating allograft antigenicity. J Reconstr Microsurg 21: 207-213, 2005.

16. Moore AM, MacEwan M, Santosa KB, Chenard KE, Ray WZ, Hunter DA, Mackinnon SE and Johnson PJ: Acellular nerve allografts in peripheral nerve regeneration: A comparative study. Muscle Nerve 44: 221-234, 2011

17. Salonen V, Aho H, Röyttä M and Peltonen J: Quantitation of Schwann cells and endoneurial fibroblast-like cells after experimental nerve trauma. Acta Neuropathol 75: 331-336, 1988

18. Hudson TW, Zawko S, Deister C, Lundy S, Hu CY, Lee K and Schmidt CE: Optimized acellular nerve graft is immunologically tolerated and supports regeneration. Tissue Eng 10: 1641-1651, 2004.

19. Gulati AK and Cole GP: Nerve graft immunogenicity as a factor determining axonal regeneration in the rat. J Neurosurg 72: 114-122, 1990.

20. Gulati AK: Immune response and neurotrophic factor interactions in peripheral nerve transplants. Acta Haematol 99: 171-174, 1998.

21. Whitlock EL, Tuffaha SH,Luciano JP, Yan Y,Hunter DA, Magill CK, Moore AM, Tong AY, Mackinnon SE and Borschel GH: Processed allografts and type I collagen conduits for repair of peripheral nerve gaps. Muscle Nerve 39: 787-799, 2009.

22. Sondell M, Lundborg G and Kanje M: Regeneration of the rat sciatic nerve into allografts made acellular through chemical extraction. Brain Res 795: 44-54, 1998.

23. Hayashi A, Moradzadeh A, Tong A, Wei C, Tuffaha SH, Hunter DA, Tung TH, Parsadanian A, Mackinnon SE and Myckatyn TM: Treatment modality affects allograft-derived Schwann cell phenotype and myelinating capacity. Exp Neurol 212: 324-336, 2008.

24. Fu SY and Gordon T: The cellular and molecular basis of peripheral nerve regeneration. Mol Neurobiol 14: 67-116, 1997.

25. Roomi MW, Ishaque A, Khan NR and Eylar EH: The PO protein. The major glycoprotein of peripheral nerve myelin. Biochim Biophys Acta 536: 112-121, 1978.

26. Fox IK, Schwetye KE, Keune JD, Brenner MJ, Yu JW, Hunter DA, Wood PM and Mackinnon SE: Schwann-cell injection of cold-preserved nerve allografts. Microsurgery 25: 502-507, 2005.

27. Hess JR, Brenner MJ, Fox IK, Nichols CM, Myckatyn TM, Hunter DA, Rickman SR and Mackinnon SE: Use of cold-preserved allografts seeded with autologous Schwann cells in the treatment of a long-gap peripheral nerve injury. Plast Reconstr Surg 119: 246-259, 2007.

28. Lee AC, Yu VM, Lowe JB III, Brenner MJ, Hunter DA, Mackinnon SE and Sakiyama-Elbert SE: Controlled release of nerve growth factor enhances sciatic nerve regeneration. Exp Neurol 184: 295-303, 2003.

29. Otto D, Unsicker K and Grothe C: Pharmacological effects of nerve growth factor and fibroblast growth factor applied to the transectioned sciatic nerve on neuron death in adult rat dorsal root ganglia. Neurosci Lett 83: 156-160, 1987.

30. Zhang Y, Luo H, Zhang Z, Lu Y, Huang X, Yang L, Xu J, Yang W, Fan X, Du B, et al: A nerve graft constructed with xenogeneic acellular nerve matrix and autologous adipose-derived mesenchymal stem cells. Biomaterials 31: 5312-5324, 2010.

31. Accioli-De-Vaconcellos ZA, Kassar-Duchossoy L and Mira JC: Long term evaluation of experimental median nerve repair by frozen and fresh nerve autografts, allografts and allografts repopulated by autologous Schwann cells. Restor Neurol Neurosci 15: 17-24, 1999.

32. Frerichs O,Fansa H, Schicht C, Wolf G, Schneider W and Keilhoff G: Reconstruction of peripheral nerves using acellular nerve grafts with implanted cultured Schwann cells. Microsurgery 22: 311-315, 2002.

33. Fraher J and Dockery P: A strong myelin thickness-axon size correlation emerges in developing nerves despite independent growth of both parameters. J Anat 193: 195-201, 1998.

34. Jia H, Wang Y, Tong XJ, Liu GB, Li Q, Zhang LX and Sun XH: Sciatic nerve repair by acellular nerve xenografts implanted with BMSCs in rats xenograft combined with BMSCs. Synapse 66: 256-269, 2012.

35. Wood MD, Kemp SW, Liu EH, Szynkaruk M, Gordon T and Borschel GH: Rat-derived processed nerve allografts support more axon regeneration in rat than human-derived processed nerve xenografts. J Biomed Mater Res A 102: 1085-1091 2014.

36. Kvist M, Sondell M, Kanje M and Dahlin LB: Regeneration in, and properties of, extracted peripheral nerve allografts and xenografts. J Plast Surg Hand Surg 45: 122-128, 2011. 